\title{
The effect of inclined position on stone free rates in patients with lower caliceal stones during SWL session
}

\author{
Basri Cakiroglu ${ }^{1}$, Orhun Sinanoglu ${ }^{2}$, Tuncay Tas ${ }^{3}$, Ismet Aydin Hazar ${ }^{3}$, Mustafa Bahadir Can Balci ${ }^{3}$ \\ ${ }^{1}$ Hisar Intercontinental Hospital Department of Urology, Istanbul, Turkey; \\ ${ }^{2}$ Maltepe University Medical School, Department of Urology, Istanbul, Turkey; \\ ${ }^{3}$ Taksim Training and Research Hospital, Department of Urology, Istanbul, Turkey.
}

\begin{abstract}
Summary Objective: To compare the outcomes of shock wave lithotripsy (SWL) combined with inclined position and SWL alone in patients with lower pole calyx stones.

Methods: Seven hundred forty patients who underwent SWL treatment for lower pole renal stones with a total diameter of $2 \mathrm{~cm}$ or less were prospectively randomized into two groups. They were comparable in terms of age, sex, and stone diameters. Patients with lower calyceal stones $(4-20 \mathrm{~mm})$ were randomized to SWL (368 patients) or SWL with simultaneous inclination (372 patients) with 30 o head down Trendelenburg position). Shock wave and session numbers were standardized according to stone size. Additional standardized shock waves were given to patients with stone fragments determined by kidney urinary bladder film and ultrasound at weeks 1, 4, 10. Results: The overall stone free rate (SFR) was 73\% (268/368) in patients with SWL alone and 81\% (300/372) in SWL with inclination at the end of $12^{\text {th }}$ week $(p=0.015)$. No significant adverse events were noted in both treatment groups.

Conclusion: Simultaneous inclination of patients during SWL session increase SFR in lower caliceal stones signifcantly compared to SWL treatment alone.
\end{abstract}

KEY WORDS: Lower pole calyx stones; Shock wave lithotripsy; Inclined position; Stone free rate.

Submitted 25 September 2014; Accepted 30 November 2014

\section{INTRODUCTION}

The prevalence of kidney stones is $2 \%$ to $3 \%$ in the general population), but the relative incidence of lower calyceal stones remains unclear (1). Shock wave lithotripsy (SWL) is widely accepted as the gold standard treatment for kidney stones less than $2 \mathrm{~cm}$ diameter. It is a non-invasive procedure, requires minimum anaesthesia, and with high patients preference. The incidence of lower calyceal kidney stones treated with SWL has risen as time passed after the primitive SWL devices came into use (2). Afterwards various studies have suggested the use SWL for these stones (2-5), However, although the clearance of lower calyceal stones after SWL seems difficult, SWL still continues to be the first choice therapy for patients with lower calyceal stones of any sizes as it is the non-invasive and easily available treatment option $(6,7)$. Investigators tried to increase stone-free rates in lower calyceal stones with some maneuvers, such as controlled inversion therapy with percussion after SWL or irrigation during treatment $(4,5)$. When outcomes of these auxiliary procedures accompanying SWL were classified by stone size ( $<1 \mathrm{~cm}$ diameter, $1-2 \mathrm{~cm}$, and $>2 \mathrm{~cm}$ ), stonefree rates after SWL treatment were found $74 \%, 56 \%$ and $33 \%$ respectively (8). Contrast opinions also were reported in other studies claiming that controlled inversion therapy did not improve results for people who underwent shock wave lithotripsy for lower pole kidney stones, among them diuresis and inversion did not significantly improve stone free rates in a series $(4,9)$.

Despite several studies supporting the benefit of auxiliary procedures, there is limited evidence suggesting that percussion, diuresis and especially inversion therapy may be beneficial for patients with lower calyceal stones after shock wave lithotripsy. Therefore, we compared the effectiveness of inclination to regular position during SWL for eliminating lower calyx calculi. We suggest that a $30^{\circ}$ head down inclination would overcome the gravitational effect of lower pole calyx that will eliminate residual fragments than usual session alone at 3 months without altering confort or hemodynamics of the patient.

\section{MATERIALS AND METHOdS}

Seven hundred forty patients between August 2006October 2013 who underwent SWL treatment for radiologically identified lower pole radiopaque renal calculi with a total diameter of $2 \mathrm{~cm}$ or less were prospectively randomized into two groups. The study protocol was reviewed and approved by the institutional ethics committee. Participants were enrolled if they were 18 years of age or older having solitary renal calculi between 4 and $20 \mathrm{~mm}$, and gave consent to randomization. Exclusion criteria were non lower calyceal stones of the same side, renal anatomical deformities such as urethral stricture or ureteropelvic junction obstruction, concomitant distal 
obstruction, renal insufficiency or gross hydronephrosis of the affected kidney, pregnancy, bleeding diathesis, significant cardiac conditions that does not permit head down inclination or uncontrolled hypertension. All subjects included into this single-blind study were prospectively randomized to control (SWL alone) or SWL with inversion therapy group. SWL was performed with Storz Modulite Fx by the 2 attending urologist using real time ultrasound for stone localization. Treatment was initiated at $14 \mathrm{kV}$, and the energy gradually increased between 20 and $24 \mathrm{kV}$, depending on the maximum level that the patient could tolerate. The numbers of shock waves (SW) used were determined by calyceal stone sizes; 4-10 mm stones (1500 SW), 11-15 mm (2000 SW), and 16$20 \mathrm{~mm}$ (2500 SW). Patients randomized to the study group had SWL at $30^{\circ}$ head down in Trendelenburg position. Patient reassessment was performed immediately at weeks 1, 4, 10, and 6 months after SWL therapy, with an evaluation using plain film of the kidney, ureter, and bladder and ultrasonography. Cases were accepted as SF if there were no radiological and ultrasonographic evidence of stone as confirmed by a blinded radiologist and validated by another blinded radiologist to provide consistency in reports. Stone free status was defined as having no visible residual stone. If the patient had significant residual fragments, SWL was repeated, with 1000 SW delivered. Complications during and after treatment were recorded. Categorical variables were compared with chi-square analysis or Fisher's exact test, and continuous variables were compared using t test.

\section{RESULTS}

The clinical characteristics of the subjects are summarized in Table 1. No significant differences were found between the 2 treatment modalities in terms of gender and age. There were not any significant difference among SWL parameters, such as stone size, session number of SWL or schock waves. The overall stone free rate (SFR) was 73\% (268/368) in patients with SWL alone and 81\% (300/372)

\section{Table 1.}

Clinical characteristics of treatment groups in the study.

\begin{tabular}{|l|c|c|c|}
\hline & Inclined & Control & p value \\
\hline Stone size & 12.65 & 12.71 & 0.95 \\
\hline Age mean & 35.1 & 36.25 & 0.93 \\
\hline SW session mean & 2.36 & 2.68 & 0.10 \\
\hline SW number & 7546 & 8982 & 0.08 \\
\hline Gender f/m & $68 / 304$ & $100 / 268$ & 0.16 \\
\hline
\end{tabular}

Table 2.

Distribution of outcomes according to treatment groups.

\begin{tabular}{|l|c|c|c|}
\hline & Stone-free & No stone-free & Total \\
\hline Inclined & $300(41 \%)$ & $72(10 \%)$ & $372(50 \%)$ \\
\hline Control & $268(36 \%)$ & $100(14 \%)$ & $368(50 \%)$ \\
\hline Total & $568(77 \%)$ & $172(23 \%)$ & $740(100 \%)$ \\
\hline
\end{tabular}

Figure 1.

Outcomes in the treatment groups at the end of $12^{\text {th }}$ week. Stone free rates in the inclined SWL

and SWL alone groups were 300/372 (81\%)

and $268 / 368(73 \%)$ respectively $(p=0.015)$.

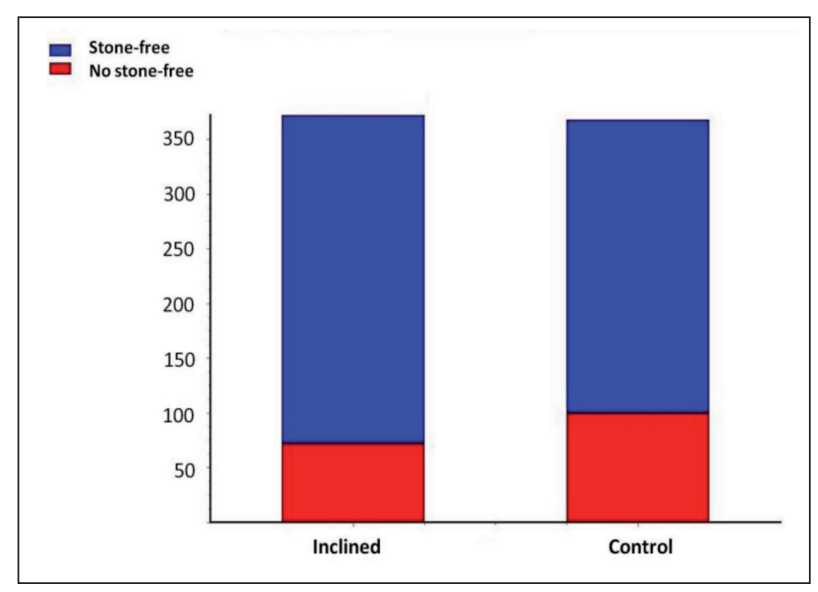

in SWL with inclination at the end of 6th month $(\mathrm{p}=$ 0.015 ) (Table 2, Figure 1). No significant adverse events were noted in both treatment arms. Retreatment and complemetary procedure rates and the duration of treatment were also not significantly different between groups. Complications were rare and occurred in 8 patients. Three subjects had steinstrasse, two had urinary tract infections whereas hematoma was seen in one patient which subsequently resolved with conservative measures.

\section{Discussion}

Achieving clearance for renal lower calyceal stones after SWL is a difficult task. Treatment of these stones by SWL does not have brillant success. Despite the adequate fragmentation the gravity force hold the fragments in the lower calyx having a reverse angle. Therefore it has been claimed that the use of inversion therapy, mechanical percussion, and administration of diuretics might enhance the stone free rates in lower calyceal calculi (10). Brownlee in 1990 suggested in his report that multiple sessions of inversion therapy could possibly have a beneficial role in the clearance of stone fragments in gravity-dependent calices following SWL (11).

However, the previous studies in 90's were retrospective in nature with limited sample sizes, and their data were methodologically flawed. Honey reported in 2008 the SWL outcomes of lower pole kidney stones at least two weeks after shock wave lithotripsy. In the series, patients were placed in a prone Trendelenberg position at $60^{\circ}$ to $70^{\circ}$, received furosemide $20 \mathrm{mg}$ IV immediately before therapy, and underwent 10 minutes of flank percussion using a chest physiotherapy device. Their results suggest the aid of the method in stone fragments passage (12). When comparing the effects of adjunctive therapy such as inversion, percussion, or hydration, only 3 prospective randomized studies were found. The first study was done by Pace et al. in which a regimen of mechanical percussion, inversion, and diuresis was compared with observation in patients with lower calyceal stones 3 months after 
SWL. Treatment outcomes were with a higher SFR versus observation (40\% vs 3\%) (5). Chiong et al. conducted a similar study with encouraging results in which they inclined their patients $45^{\circ}$ angle with percussion, forced hydration and diuresis following SWL sessions. The SFR was $62 \%$ in treatment arm whereas success rate was 35\% in observed patients (8). In the most recent prospective study of Wing Seng Leong et al. the evidence suggest that SWL with simultaneous inversion is a valuable combination in assisting the passage of lower pole renal stones with a SFR of $76 \%$, despite the lack of significance. The number of their participants were 140. Stone size was found to affect the results of the overall SFRs after SWL of lower calyceal stones. Authors found out that SFRs were 79\% and $69 \%$ for stones $<10 \mathrm{~mm}$ and 11 to $20 \mathrm{~mm}$ respectively (10). In our study a total number of 740 were prospectively randomized into two groups. We applied SWL sessions only with concomitant $30^{\circ}$ inclination without using $45^{\circ}$ to $60^{\circ}$ inversion, percussion and forced diuresis unlike the previous reports. The adoption of these exagerated auxiliary methods seems to be disappointing because they require additional effort by the doctors and patients during and after SWL. There are limitiations to our study; first, we did not stratified the SFR rates according to stone sizes, second, lack of data on stone composition or density and skin to stone distance.

Lastly, it is also worth noting that although several above mentioned studies support the adjunctive methods in lower calyceal SWL treatment, some other authors claimed in the past that there is no benefit of these auxiliary procedures. Among these, Rodrigues et al. reported outcomes of the study group who received adjunctive therapy following shock wave lithotripsy of oral hydration, $60^{\circ}$ to $75^{\circ}$ inversion, and percussion. Stone-free status was achieved in $84 \%$ of participants who did not receive adjunctive therapy, in contrast to $64.7 \%$ SFR in those undergoing adjunctive therapy. Authors concluded that inversion therapy did not ameliorate outcomes of SWL for lower calyceal stones (9).

In conclusion, the overall SFR at 6 months after SWL in $30^{\circ}$ inclined position without usage of forced hydration and/or percussion was $81 \%$ vs. $73 \%$ of the control group in the present study. Therefore we recommend that inclination should be used for lower pole calyx renal calculi because it provides an improved clearance with both minimal patient cooperation and no additional effort of the doctor.

\section{Competing interests}

The authors declare that they have no competing interests.

\section{AUTHORS' CONTRIBUTIONS}

1. Study concept and design: BC; 2. Acquisition of data: BC, OS; 3. Drafting of the manuscript: MBCB, TT; 4. Critical revision of the manuscript for important intellectual content: IH; 5. Statistical analysis: OS; 6. Administrative, technical, and material support: BC, IH, 7. Study supervision: OS; 8. Design of the generator and probe and acquisition of data relating to the functioning of the apparatus: BC, TT. All authors read and approved the final manuscript.

\section{REFERENCES}

1. Poch M, Haleblian GE. Minimally invasive stone surgery: percutaneous, ureteroscopic and extracorporeal approaches to renal and ureteral calculi. Medicine E Health, Rhode Island. 2009; 92:339-41.

2. Lingeman JE, Siegel YI, Steele B, et al. Management of lower pole nephrolithiasis: a critical analysis. Journal of Urology. 1994; 151:663-7.

3.Albala DM, Assimos DG, Clayman RV, et al. Lower pole I: a prospective randomized trial of extracorporeal shock wave lithotripsy and percutaneous nephrostolithotomy for lower pole nephrolithiasis-initial results. J Urol. 2001; 166:2072-80.

4. Albanis S, Ather HM, Papatsoris AG, et al. Inversion, hydration and diuresis during extracorporeal shock wave lithotripsy: does it improve the stone-free rate for lower pole stone clearance? Urol Int. 2009; 83:211-216.

5. Pace KT, Tariq N, Dyer SJ, et al. Mechanical percussion, inversion and diuresis for residual lower pole fragments after shock wave lithotripsy: a prospective, single blind, randomized controlled trial. J Urol. 2001; 166:2065-2071.

6. Kekre NS, Kumar S. Optimizing the fragmentation and clearance after shock wave lithotripsy. Current Opinion in Urology. 2008; 18:205-9

7. Raman JD, Pearle MS. Management options for lower pole renal calculi. Current Opinion in Urology. 2008; 18:214-9.

8. Chiong E, Hwee ST, Kay LM, et al. Randomized controlled study of mechanical percussion, diuresis, and inversion therapy to assist passage of lower pole renal calculi after shock wavelithotripsy. Urology. 2005; 651070-4.

9. Rodrigues Netto N Jr, Claro JF, Cortado PL, Lemos GC. Adjunct controlled inversion therapy following extracorporeal shock wave lithotripsy for lower pole caliceal stones. Journal of Urology. 1991; 146:953-4.

10. Leong WS, Liong ML, Liong YV, et al. Does simultaneous inversion during extracorporeal shock wave lithotripsy improve stone clearance: a long-term, prospective, single-blind, randomized controlled study.Urology. 2014; 83:40-4.

11. Brownlee N, Foster M, Griffith DP, Carlton CE Jr. Controlled inversion therapy: an adjunct to the elimination of gravity-dependent fragments following extracorporeal shock wave lithotripsy. J Urol. 1990; 143:1096-8.

12. Honey J. Treating lower pole renal stones: in defence of shock wave lithotripsy. Canadian Urological Association Journal. 2008; 2:625-7.

\section{Correspondence}

Basri Cakiroglu, MD (Corresponding Author)

drbasri@gmail.com

Hisar Intercontinental Hospital, Saray Mah. Siteyolu Cad.No:7

34768 Umraniye, Istanbul, Turkey

Orhun Sinanoglu, MD

orhundr@hotmail.com

Maltepe University Medical School, Department of Urology, Maltepe, Istanbul, Turkey

Tuncay Tas, MD

tastuncay@gmail.com

Aydin Ismet Hazar, MD

mdhazar@hotmail.com

Mustafa Bahadir Can Balci, MD

drbalci@yahoo.com

Taksim Training and Research Hospital, Department of Urology, Gaziosmanpasa, Istanbul, Turkey 